S. Mak-Kregar · F. J. M. Hilgers · P. C. Levendag - J. J. Manni · H. Lubsen

J. L. N. Roodenburg - J. M. H. van der Beek · A. G. L. van der Meij

\title{
A nationwide study of the epidemiology, treatment and survival of oropharyngeal carcinoma in the Netherlands
}

Received: 2 September 1994 / Accepted: 7 December 1994

\begin{abstract}
Seven head and neck oncology cooperative groups in the Netherlands have reviewed the epidemiology, staging, treatment and survival of oropharyngeal
\end{abstract}

Presented at the combined meeting of the Society of Head and Neck Surgeons and the European Organization for Research and Treatment of Cancer, Paris, France, 25-28 May 1994

S. Mak-Kregar

Comprehensive Cancer Center, Amsterdam, The Netherlands

F. J. M. Hilgers (凶)

Department of Otolaryngology-Head and Neck Surgery,

The Netherlands Cancer Institute, Plesmanlaan 121,

NL-1066 CX Amsterdam, The Netherlands

F. J. M. Hilgers

Department of Otolaryngology, University Hospital, Amsterdam, The Netherlands

P. C. Levendag

Department of Radiotherapy, Dr. Daniel Den Hoed Cancer Center, Rotterdam, The Netherlands

P. C. Levendag

Department of Otolaryngology, University Hospital,

Rotterdam, The Netherlands

J. J. Manni

Department of Otolaryngology, University Hospital, Nijmegen, The Netherlands

H. Lubsen

Department of Otolaryngology, University Hospital, Utrecht, The Netherlands

J. L. N. Roodenburg

Department of Maxillofacial Surgery, University Hospital,

Groningen, The Netherlands

J. M. H. van der Beek

Department of Otolaryngology, University Hospital,

Maastricht, The Netherlands

J. M. H. van der Beek

Institute of Radiotherapy, Limburg, The Netherlands

A. G. L. van der Meij

Department of Otolaryngology, University Hospital, Leiden, The Netherlands

Present address:

${ }^{1}$ Department of Otolaryngology, University Hospital,

Maastricht, The Netherlands carcinoma patients treated between 1986 and 1990. In all, 640 patients with squamous cell carcinoma (628, $98 \%)$ or undifferentiated carcinoma $(12,2 \%)$ referred for primary treatment were analyzed. The total group included 441 males $(69 \%)$ and 199 females $(31 \%)$, with a median age of 59 years (range, 30-92). Tumor distribution by subsite was the tonsillar region ( 372 patients, $58 \%$ ), base of the tongue/vallecula $(179,28 \%)$, soft palate/uvula $(62,10 \%)$ and posterior oropharyngeal wall $(27,4 \%)$. Forty-four patients $(7 \%)$ had stage I disease, $106(17 \%)$ had stage II disease, $157(24 \%)$ stage III, and $319(50 \%)$ stage IV. Staging was unknown in 14 patients ( $2 \%$ ). Radiotherapy was given to the primary tumor in 408 patients $(64 \%)$, surgery and radiotherapy to 147 (23\%), surgery alone to $42(7 \%)$, other treatments to 14 (2\%) and no treatment to 29 patients (4\%). The 5-year overall survival was $28 \%$ and the 5-year disease-specific survival was $41 \%$. This latter survival was $35 \%$ in males and $51 \%$ in females $(P=0.003)$. Five-year survival by subsite was $54 \%$ in the soft palate/uvula, $42 \%$ in the tonsillar region, $33 \%$ in the base of the tongue and $32 \%$ in the posterior oropharyngeal wall $(P=0.003)$. When analyzing survival by stage, 5 -year survival in patients with stage I disease was $68 \%$ and decreased significantly to $27 \%$ in stage IV disease $(P<0.001)$. Best survival occurred in patients treated with surgery alone $(80 \%)$, was less in the group treated by surgery and radiotherapy $(51 \%)$, and decreased further in patients treated by radiotherapy alone $(36 \%)(P<0.0001)$. By multivariate analysis, stage, sex and midline localization were found to be significant prognostic factors.

Key words Oropharyngeal carcinoma - Epidemiology Staging $\cdot$ Treatment $\cdot$ Survival

\section{Introduction}

Oropharyngeal carcinoma is in general a low incidence tumor, accounting for $0.3-0.5 \%$ of all malignancies [11, 13 ] and predominantly affecting men in the sixth and sev- 
enth decades of life $[3,8,20]$. Since tumors usually are asymptomatic during their growth, most patients present with advanced tumors, i.e. stages III-IV of the 1987 UICC system [22].

In the absence of convincing results from comparative studies optimal management is subject to an ongoing debate. In general, patients with local or locoregional disease are offered treatment with a curative intent, being the case in approximately $80 \%$ of all patients [18]. The remainder, suffering from disseminated disease and/or being in poor general condition, receive palliative treatment or no treatment at all. The main modalities of treatment with curative intent are surgery and radiotherapy, applied either as single modalities, often in stages I-II, or in combination (stages III-IV).

When comparing results of treatment, 5-year overall survival rates of approximately $40 \%$ are regularly obtained without striking differences between treatment modalities: [4, 7, 9-11, 23, 24]. However, occasional series have reported poorer $(15-20 \%)[15]$ or better $(62-64 \%)[1,16]$ results. In these cases, the leading prognostic factor has been the extension of disease on admission [7, 17]. However, in predicting survival sex, age, general condition, tumor subsite, response to treatment and the presence or absence of second primary neoplasms also need to be considered, as they may significantly influence prognosis.

In order to answer questions about various factors affecting malignancy, the Dutch Head and Neck Cooperative Group has conducted a nationwide study in order to address issues of epidemiology, treatment and survival of patients with oropharyngeal carcinoma.

\section{Materials and methods}

Seven leading medical centers that participate in the Dutch Head and Neck Cooperative Group, i.e. the University Hospitals of Groningen, Leiden and Maastricht in combination with the Radiotherapeutic Institute of Limburg, Nijmegen and Rotterdam in cooperation with the Dr. Daniel Den Hoed Cancer Center, Utrecht and The Netherlands Cancer Institute in combination with the University of Amsterdam, have participated in the present project. In the preparatory phase, feasibility and scope of the study, time schedule and practical aspects were examined using previous experiences of the Cooperative Group [19]. Next, a checklist and related manual were designed for the purposes of the study. Each following step was taken upon reaching a consensus of the overall project design.

\section{Data collection}

In each participating center the charts of patients admitted between 1986 and 1990 for treatment of oropharyngeal carcinoma were reviewed. Data related to epidemiology, staging, treatment and survival were collected using standardized checklists and returend as hard copy to the Comprehensive Cancer Center, Amsterdam (IKA). There, a centralized data entry using a scientific information retrieval (SIR) database, version 3.2, was performed. Patients who were admitted to more than one center were identified by cross-checking of epidemiological data, and totals were adjusted accordingly.

\section{Patients}

From 694 patient forms returned, 22 were excluded because of multiple admissions, 18 due to other tumor histologies and 8 for exceeding the study period. Additionally, 6 patients were receiving treatment for recurrent disease and were also excluded from the present review. Thus, 649 patients admitted for primary treatment of histologically proven squamous cell carcinoma (628 patients) or undifferentiated carcinoma (12 patients) of the oropharynx were analyzed. Among the total group, 441 patients $(69 \%)$ were males and $199(31 \%)$ females, with a median age of 59 years (range, 30-92 years).

Patients were followed for at least 3 years, or until death, and were censored if they were lost to follow-up before the end of that period. Overall survival was defined as the time between the date of diagnosis and the end of follow-up or death, regardless of tumor status. In calculating disease-specific survival, only those patients who died with local, regional and/or distant disease from oropharyngeal tumor were considered.

\section{Statistical analysis}

Survival curves were calculated using the life table method. Disease-specific survival was tested as a more appropriate indicator for mortality due to oropharyngeal carcinoma. Possible bias was examined by using Pearson's chi-squared test to evaluate potential differences in assessment of tumor status at death at each participating center.

In the univariate analysis of disease-specific survival, the $\log$ rank test was applied. Single prognostic factors were tested in Cox's [2] proportional hazard model with stepwise backward elimination.

Detailed analysis of locoregional recurrence rates in general and in the specific subsites will be published elsewhere. In this paper analysis of all patients with respect to treatment and survival will be presented.

\section{Results}

\section{Subsite localizations of tumor}

Of the 640 oropharyngeal carcinomas, $372(58 \%)$ originated from the tonsillar region, $179(28 \%)$ from the base of the tongue, $62(10 \%)$ from the soft palate/uvula and 27 (4\%) from the posterior oropharyngeal wall.

Five hundred and sixty-seven tumors $(89 \%)$ originated from the lateral parts of the oropharynx, and $73(11 \%)$ from the midline. Midline lesions encompassed 33 base of tongue tumors (18\% of all tumors in this subsite), 11 posterior wall lesions (41\% of all), 27 soft palate/uvula neoplasms (43\%) and 2 tonsillar region tumors that were grossly exceeding the midline. The prevalent side was left in 269 patients (42\%) and right in 298 patients $(47 \%)$.

\section{Staging}

UICC classifications and staging system from 1978 [21] and 1987 [22] were recorded. Distribution by tumor $(\mathrm{T})$ was identical in both classifications and was as follows: T1, 79 (12\%); T2, $197(31 \%)$; T3, 196 (31\%); T4, 165 $(26 \%)$. The T stage was unknown in 3 patients. Using the 1978 UICC classification, distribution by node (N) status 


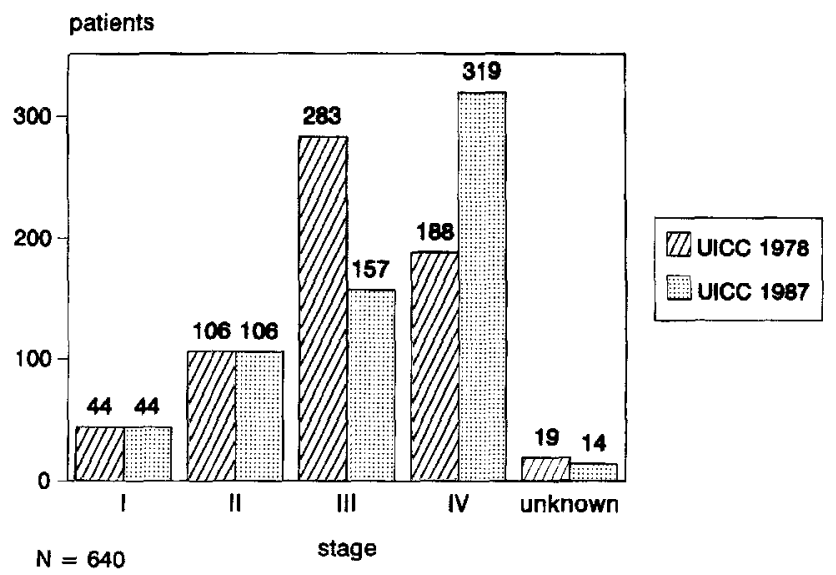

Fig. 1 Case distribution of oropharyngeal carcinoma by stage (UICC 1978) and (UICC 1987)

was: N0, $258(40 \%)$; N1, $235(37 \%)$; N2, $72(11 \%)$; N3, $67(11 \%)$. Nodal status was unknown in 8 patients $(2 \%)$. When using the 1987 classification for $\mathrm{N}$ disease, 151 cases were N1 (24\%), 182 were N2 (28\%) and 46 were N3 $(7 \%)$. The vast majority of patients $(620,94 \%)$ was free of distant metastasis on admission, while 9 patients $(1 \%)$ had metastases and status for metastasis could not be determined in 29 patients (5\%).

Distribution by stage using the 1978 UICC classification was: 44 patients (7\%) stage I; 106 patients (17\%) stage II; 283 patients (44\%) stage III; 188 patients (29\%) stage IV; 19 patients (3\%) with an unknown stage of the disease. With the 1987 UICC classification, 44 patients (7\%) had stage I disease, 106 (17\%) stage II disease, 157 (24\%) stage III disease and $319(50 \%)$ stage IV disease (Fig. 1).

\section{Treatment}

Of 640 patients, $500(78 \%)$ were treated with intention to cure, $128(20 \%)$ received palliative treatment and in the remaining 12 patients $(2 \%)$ the intention of treatment was not clear in retrospect. The rate of patients who received palliation increased with the stage of the disease, and was $2 \%$ in stage I and $34 \%$ in stage IV.

Treatment corresponded to standard protocols in each center in 530 patients $(83 \%)$ and was deviant from the standard schedules in $98(15 \%)$. Adherence to protocol was not clear in retrospect for 12 patients $(2 \%)$. The reasons to deviate from the standards were: poor general condition ( 26 patients, $4 \%$ ), patient's choice ( 20 patients, $3 \%$ ), second primary tumors (15 patients, $2 \%$ ), participation in EORTC 22791 trial [6] (9 patients, 1\%), disease progression during radiotherapy ( 6 patients, $1 \%$ ), very advanced tumors ( 5 patients, $1 \%$ ) and unknown site of primary tumor at the beginning of treatment ( 3 patients). Interestingly, high age was reported as the single reason for deviation from standard treatment in only 1 patient (83 years of age).

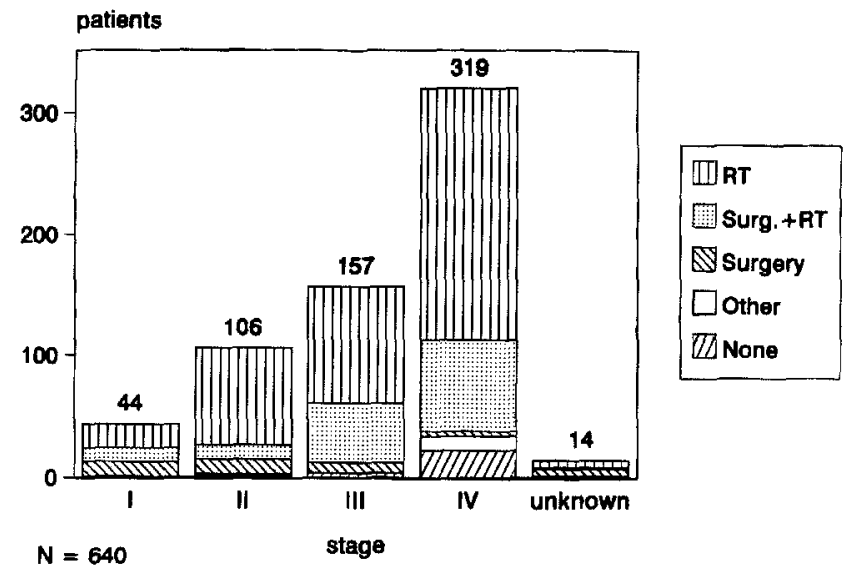

Fig. 2 Treatment modality for oropharyngeal carcinoma by stage (UICC 1987)

\section{Treatment modalities}

Globally, treatment of the primary site consisted of radiotherapy in 408 patients (64\%), surgery and radiotherapy in 147 patients $(23 \%)$, surgery by wide local or composite resections in 42 patients $(7 \%)$ and other forms of treatment (including chemotherapy) in 14 patients (2\%). In total, neck node resections were performed in 217 patients (34\%): radical (142 patients, $22 \%$ ), modified (58 patients, $9 \%$ ) or selective (17 patients, 3\%). Twenty-nine patients $(4 \%)$ were not treated at all. Treatment modality by stage is shown in Fig. 2. A group of 63 patients who received radiotherapy to their primary tumors in combination with surgical treatment of the regional neck nodes were considered to be treated with radiotherapy alone for the purpose of this report.

\section{Vital status}

All figures related to vital status and survival present "5-year" results (Table 1). Split up by center, some differences in tumor status at death were observed, but these are not likely to cause appreciable bias when disease-specific survival was analyzed rather than overall survival. The observed differences may well have been caused by chance $(P=0.35)$.

Table 1 Vital status of 640 patients with oropharyngeal cancer (NED no evidence of disease)

\begin{tabular}{lrr}
\hline Clinical status & \multicolumn{2}{c}{ Patients } \\
\hline Alive, NED & 225 & $(35 \%)$ \\
Alive, with tumor & 17 & $(3 \%)$ \\
Dead, NED & 74 & $(12 \%)$ \\
Dead, with tumor & 316 & $(49 \%)$ \\
Lost to follow-up & 8 & $(1 \%)$ \\
Total & $640(100 \%)$ \\
\hline
\end{tabular}




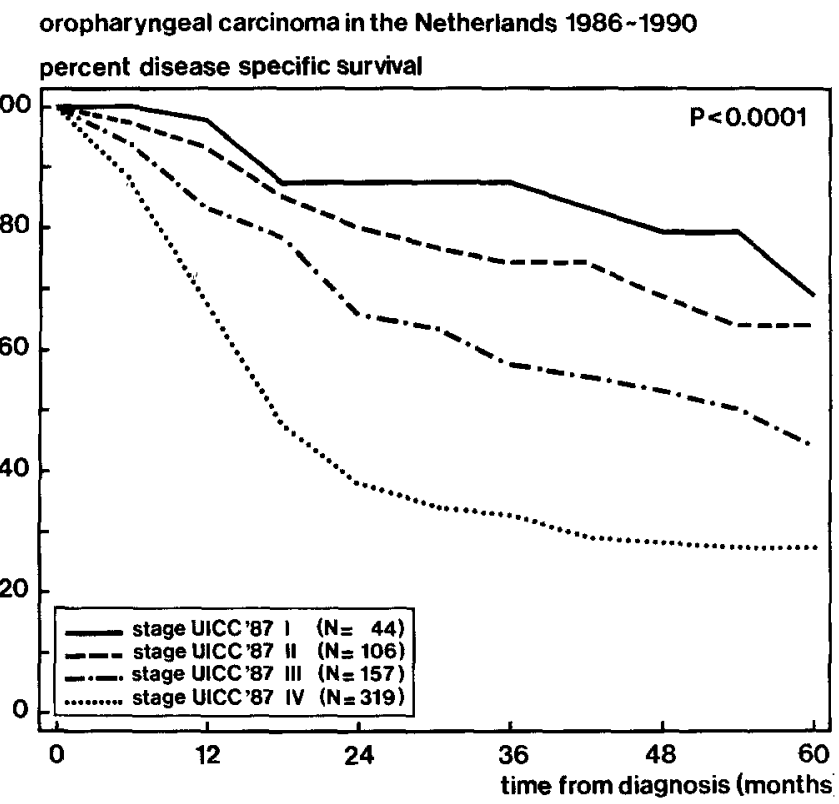

Fig. 3 Disease-specific survival by stage (UICC 1987)

\section{Survival}

Overall survival was $28 \%$ and disease-specific survival was $41 \%$. Overall survival in males was $22 \%$ and in females $39 \%(P=0.0001)$, while disease-specific survival was $35 \%$ and $51 \%$ in males and females, respectively $(P$ $=0.003$ ). Overall survival by subsite was $44 \%$ in the soft palate/uvula, $30 \%$ in the tonsillar region, $23 \%$ in the base of the tongue and $13 \%$ in the posterior oropharyngeal wall $(P=0.001)$. Corresponding figures for disease-specific survival in each site were $54 \%, 42 \%, 33 \%$ and $32 \%$, respectively $(P=0.003)$.

Overall survival per stage of disease (UICC, 1987) was $50 \%$ in stage I and II, $28 \%$ in stage III and $16 \%$ in stage IV $(P<0.0001)$. As shown in Fig. 3, disease-specific survival for stage I disease was $68 \%$ and decreased significantly to $27 \%$ for stage IV disease $(P<0.0001)$.

Disease-specific survival in patients treated by surgery was $80 \%$, surgery and radiotherapy $51 \%$, radiotherapy alone $36 \%$, and treatment by other modalities $7 \%(P<$ 0.0001 ). Treatment including surgery (alone or in combination with radiotherapy) was associated with a higher disease-specific survival than radiotherapy alone: stage I, $68 \%$ vs $34 \%$; stage II, $81 \%$ vs $41 \%$; stage III, $36 \%$ vs $25 \%$; stage IV, $31 \%$ vs $14 \%(P<0.0001)$.

When split up by center, a difference in disease-specific survival was seen at 5 years, ranging from $24 \%$ to 64\% $(P=0.009)$.

\section{Univariate analysis}

From analysis of disease-specific survival the following factors emerged as univariately statistically significant: sex $(P=0.003)$, age $(P=0.013)$, T status $(P<0.0001)$, N status $(P<0.0001)$, stage UICC $1987(P<0.0001)$, sub-
Table 2 A revised staging system for oropharyngeal cancer

\begin{tabular}{clll}
\hline I & T1-2 & N0-1 & M0 \\
II & T1-2 & N2 & M0 \\
& T3 & N0-1 & M0 \\
& T4 & N0 & M0 \\
III & T1-2 & N3 & M0 \\
& T3 & N2 & M0 \\
& T4 & N1 & M0 \\
IV & T3 & N3 & M0 \\
& T4 & N2-3 & M0 \\
& Any T & Any N & M1 \\
\hline
\end{tabular}

Table 3 Multivariate analysis of disease-specific survival for oropharyngeal cancer

\begin{tabular}{lll}
\hline Factor & $P^{\mathrm{a}}$ & $P^{\mathrm{b}}$ \\
\hline Sex & $=0.015$ & $=0.006$ \\
Age & $=0.006$ & $=0.084$ \\
Midline origin & $<0.0001$ & $<0.0001$ \\
Revised stage & $<0.0001$ & $<0.0001$ \\
Treatment modality & $<0.0051$ & \\
Center & $=0.21$ & $=0.23$ \\
\hline
\end{tabular}

${ }^{\text {a }}$ Controlled for all variables except for treatment modality

${ }^{\mathrm{b}}$ Controlled for all variables, including treatment modality

site involvement $(P=0.003)$, midline origin $(P<0.0001)$, treatment $(P<0.0001)$, and center $(P=0.009)$.

\section{A revised staging system}

In order to control for the prognostic factors obtained, the combination of T, N and M as proposed in the UICC stage grouping was tested. Parameters related to epidemiology, tumors and treatment available for 594 patients were entered in the proportional hazard model. In this group of patients, regrouping of the existing $\mathrm{T}, \mathrm{N}$ and $\mathrm{M}$ categories over the stages led to distribution superior to the UICC system. A detailed description of this staging system (see Table 2) will be published elsewhere [5].

\section{Multivariate analysis}

All prognostic factors obtained in the univariate analysis and the revised staging system were entered in a multivariate model. The prognostic role of the centers decreased when controlled for stage and midline origin $(P=$ 0.051 and $P=0.21$ for the two staging systems, respectively), leading to complete elimination of the prognostic value of the center when revised stage grouping was utilized.

Stage, midline origin and sex appeared to have persistent prognostic value throughout all analyses $(P<0.0001$, $P<0.0001$ and $P=0.006$, respectively).

Prognostic value of age was no longer significant after controlling for treatment $(P=0.084)$. 
With respect to treatment, adherence to the standard protocols, surgery (plus radiotherapy) as compared to radiotherapy and "other treatment" compared to no treatment were associated with better disease-specific survival $(P=0.0019,0.0051$ and 0.015 , respectively). $P$ values for the prognostic factors in the multivariate analysis are summarized in Table 3.

\section{Discussion}

In diseases with low incidence and no established treatment recommendations, reviews of large numbers of patients provide valuable insights even when different treatment modalities and/or more clinical institutes are involved. Short checklists with pragmatic questions about large numbers of patients are appropriate for obtaining sets of hard comparable data, and are feasible within a limited time span. The Dutch Head and Neck Cooperative Group has chosen this approach, realizing that some questions would remain unanswered but certain useful insights would be obtained. Earlier studies of the group have already clearly shown this [19].

The rate of missing values in our present study is remarkably low. From 1989 to 1990 , the Netherlands Cancer Registry reported findings in 447 patients with oropharyngeal carcinoma, and found that $\mathrm{T}, \mathrm{N}, \mathrm{M}$ or subsite data were missing from $8-15 \%$ in cases reviewed (source: the Netherlands Cancer Registry). Our own findding of missing information in cases reviewed was less than $5 \%$. Several reasons may be responsible for this discrepancy: quality of documentation over different hospitals, types of patients, motivation related to the study, etc. Specialized cancer centers participating in this study have more extensive and often better structured documentation on cancer patients than do general hospitals. Secondly, patients not referred to cancer centers are more likely to be old or disabled, have advanced disease and/or are not prepared to undergo rather extensive treatment. In such cases, an elaborate diagnostic work-up is often not performed and some data are invariably missing.

Our present study is estimated to have encompassed more than half of the patients with oropharyngeal carcinoma managed in the Netherlands during the period studied. With respect to age, sex and histopathology, similar distributions were observed in this patient population and the one covered by the Cancer Registry. In the case of subsites, T, N, M and stage of the disease, comparison is hampered by the discrepancies in missing values, but striking differences do not seem to emerge.

A significant influence of participating centers with regard to survival in univariate analysis was initially observed. However, when controlled for other significant prognostic factors (i.e. sex, differences in staging systems and midline origin of tumor), differences among centers decreased below the level of statistic significance. Attempts to compare one's own results with other reports are not exceptional in the literature. Nevertheless, separate studies differ in methods of selecting, staging and treating patients and/or in the extent of reporting on the various procedures employed. Even when a study methodology is standardized, striking differences may be found due to differences in patient populations and treatment. This observation emphasizes the urge for careful interpretation of data, particularly if they are obtained from separate investigators.

A comparison of treatment modalities with respect to end-results forms another pitfall. In our series superior survival rates were associated with surgery, with or without radiotherapy, above radiotherapy alone. The difference was statistically significant even when controlled for other prognostic factors, including tumor stage. However, this outcome is not supported by other published papers. Again, false-positive conclusions regarding the prognostic significance of some factors or treatment modalities in particular may be drawn in the absence of careful analysis and interpretation of all data. The role of different treatment modalities for oropharyngeal cancers managed in the Netherlands will be discussed in more detail in subsequent papers, and in particular will deal with different subsites of the oropharynx.

One outcome of our present study is the evidence for a prognostic role of midline origin of oropharyngeal tumors. Usually these tumors are split up by subsite, tonsillar carcinomas having better prognosis than base of tongue or posterior wall tumors [14]. However, the relationship between laterality of lesions and prognosis being more significant than the relation between anatomical subsite and prognosis had not been demonstrated before.

Despite the established prognostic role of disease stage, the optimal definition of stages is still subject to active research. The official committees of UICC and the American Joint Committee for Cancer Staging (AJCC) and independent authors regularly evaluate practical and prognostic aspects of current staging systems. Proposals for improvement inevitably follow, as reflected by the UICC 1987 and AJCC 1988 system [22], TANIS [12] and our revised system. The latter lead to a more balanced distribution of patients over stages and to a superior prognostic distinction when compared with earlier systems [5].

Acknowledgements On behalf of the Dutch Head and Neck Oncology Cooperative Group the authors would like to acknowledge the financial and practical support of the Schumacher Foundation, Amsterdam, the Academic Hospital Maastricht, the Head and Neck Cooperative Group Nijmegen, the Head and Neck Cooperative Group Rotterdam, The Netherlands Cancer Institute, Amsterdam, and last but not least the Comprehensive Cancer Center Amsterdam. A. A. M. Hart, MSc (statistician at the Netherlands Cancer Institute) and O. Visser, MD (research coordinator at the Comprehensive Cancer Center, Amsterdam) are sincerely thanked for their major contribution to the data acquisition, computerized data management and extensive statistical analysis.

\section{References}

1. Ang KK, Peters LJ, Maor MH, Morrison WH, Wendt CD, Brown BW (1990) Concomitant boost radiotherapy schedules in the treatment of carcinoma of the oropharynx and nasopharynx. Int J Radiat Oncol Biol Phys 19: 1339-1345 
2. Cox DR (1972) Regression models and life tables. J R Stat Soc $34: 187-220$

3. Farr HW, Goldfarb PM, Farr CM (1980) Epidermoid carcinoma of the mouth and pharynx at Memorial Sloan-Kettering Cancer Center, 1965 to 1969 . Am J Surg 140:563-567

4. Geoffray B, Luboinski P, Micheau C, Richard JM (1987) Combined treatment of cancer of the posterior oral cavity and oropharynx. Clin Otolaryngol 12:429-439

5. Hart AAM, Mak-Kregar S, Hilgers FJM, Levendag PC, Manni JJ, Spoelstra HA, Bruaset I, Laan BFAM van der, Annyas AA, Beek JMH van der, Meij AGL van der, Visser O. The importance of correct stage grouping in oncology: results of a nationwide study on oropharyngeal carcinoma in the Netherlands. Cancer (in press)

6. Horiot JC, Le Fur R, N'Guyen T, Chenal C, Schraub S, Alfonsi S, Gardani G, Van den Bogaert W, Danczak S, Bolla M, Van Glabbeke M, De Pauw M (1990) Hyperfractionated compared with conventional radiotherapy in oropharyngeal carcinoma: an EORTC randomized trial. Eur J Cancer 26:779-780

7. Hussey DH, Latourette HB, Panje WR (1991) Head and neck cancer: an analysis of the incidence, patterns of treatment, and survival at the university of Iowa. Ann Otol Rhinol Laryngol $100: 1-16$

8. IARC/IACR (1987) Cancer incidence in five continents, vol 5. IARC, Lyon

9. Jesse RH, Sugerbaker EV (1976) Squamous cell carcinoma of the oropharynx: why we fail. Am J Surg 132:435-438

10. Johansen J, Christensen PH, Jorgensen K, Andersen JE (1991) Epidermoid carcinoma of the pharynx. Acta Oncol 30:33-37

11. Johansen LV, Overgaard J, Overgaard M, Birkler N, Fisker A (1990) Squamous cell carcinoma of the oropharynx: an analysis of 213 consecutive patients scheduled for primary radiotherapy. Laryngoscope 100:985-990

12. Jones GW, Broman G, Goodyear M, Marcellus D, Hodson DI (1993) Comparison of the addition of $\mathrm{T}$ and $\mathrm{N}$ integer scores with TNM stage groups in head and neck cancer. Head Neck $15: 497-503$
13. Landelijk Overleg Kankercentra (1991) Progress report Dutch Cancer Registry 1988. LOK, Utrecht

14. Mak-Kregar S, Baris G, Lebesque JV, Balm AJM, Hart AAM, Hilgers FJM (1993) Radiotherapy of tonsillar and base of tongue carcinoma. Prediction of local control. Oral Oncol Eur J Cancer 29B : 119-125

15. Pinto LH, Canary PCV, Araujo CMM, Bacelar SC, Souhami L (1991) Prospective randomized trial comparing hyperfractionated versus conventional radiotherapy in stages III and IV oropharyngeal carcinoma. Int J Radiat Oncol Biol Phys 21:557-562

16. Rabuzzi DD, Mickler AS, Clutter DJ, Chung CT, Sagerman RH (1982) Treatment results of combined high-dose preoperative radiotherapy and surgery for oropharyngeal cancer. Laryngoscope 92: 989-992

17. Smith RR, Frazell EL, Caulk R, Holinger PH, Russell WO (1963) The American Joint Committee's proposed method of stage classification and end-result reporting applied to 1320 pharynx cases. Cancer 16:1505-1520

18. Stell PM (1976) Tumours of the oropharynx (review). Clin Otolaryngol 1:71-90

19. Terhaard CHJ, Hordijk GJ, Broek P van den, Jong PC de, Snow GB, Hilgers FJM, Annyas AA, Tjho-Heslinga RE, de Jong JMA (1992) A retrospective study of the Dutch Head and Neck Oncology Cooperative Group: study design and general results. Clin Otolaryngol 17:393-402

20. UICC (1975) Clinical oncology. Springer, Berlin Heidelberg New York, pp 136-137

21. UICC (1978) TNM Classification of malignant tumours, 3rd edn. UICC, Geneva, pp 27-32

22. UICC (1987) TNM Classification of malignant tumours, 4th edn. Springer, Berlin Heidelberg New York, pp 1-22

23. Viani L, Dammeijer P, Jones AS, Dalby JE, Stell PM (1991) Recurrence of oropharyngeal carcinoma after radiotherapy. J Laryngol Otol 105:24-28

24. Weller SA, Goffinet DR, Goode RL, Bagshaw MA (1976) Carcinoma of the oropharynx: results of megavoltage radiation therapy in 305 patients. Am J Roentgenol 126:236-247 